Meta

Journal des tradlucteurs

Translators' Journal

\title{
La traduction scientifique : un phénomène récent ?
}

\section{Henri Van Hoof}

Volume 26, numéro 3, septembre 1981

URI : https://id.erudit.org/iderudit/003087ar

DOI : https://doi.org/10.7202/003087ar

Aller au sommaire du numéro

Éditeur(s)

Les Presses de l'Université de Montréal

ISSN

0026-0452 (imprimé)

1492-1421 (numérique)

Découvrir la revue

Citer cet article

Van Hoof, H. (1981). La traduction scientifique : un phénomène récent ? Meta, 26(3), 215-222. https://doi.org/10.7202/003087ar d'utilisation que vous pouvez consulter en ligne.

https://apropos.erudit.org/fr/usagers/politique-dutilisation/ 


\section{La traduction scientifique: un phénomène récent?}

HeNRI VAN HoOF

La traduction scientifique est de date relativement récente. La traduction littéraire a existé de tous temps, la traduction juridique aussi. La traduction scientifique n'est apparue que très tard dans notre civilisation occidentale, ceci pour une raison très simple : c'est que pendant des siècles, la science s'est exprimée dans une langue universelle qui était le latin'.

Ces paroles du professeur Lépine, membre de l'Académie de médecine et de l'Académie des sciences, traduisent assez bien une opinion très répandue dans le grand public, à savoir que la traduction technico-scientifique est une création de notre temps.

De fait, plusieurs facteurs permettent d'expliquer cette conviction. Tout d'abord, il est exact que la traduction littéraire a toujours existé; elle est même la seule qui, dans les siècles passés, ait tenté les théoriciens. Tous les écrits consacrés aux problèmes de la traduction, à commencer par ceux de Dolet en France, de Tytler en Grande-Bretagne, de Schleiermacher en Allemagne, pour ne citer que les plus anciens, ont toujours porté exclusivement sur des textes littéraires. Il a fallu attendre la deuxième moitié du $\mathrm{xx}^{\mathrm{e}}$ siècle pour voir paraître le premier ouvrage consacré aux problèmes spécifiques de la traduction non littéraire $^{2}$. On comprend qu'une telle situation soit de nature à faire considérer la traduction technico-scientifique comme une création récente. Les statistiques semblent d'ailleurs abonder dans ce sens. Si l'on s'en réfère aux chiffres de l'Index Translationum, ce répertoire de l'UNESCO dont chaque volume reprend les traductions publiées annuellement dans le monde, on constate que, jusqu'en 1962, plus de la moitié des œuvres traduites relèvent du domaine littéraire. Ce n'est qu'à partir de 1963 que la courbe s'inverse et que l'on voit les traductions scientifiques prendre le pas sur les traductions littéraires. Tous ces faits, tous ces chiffres semblent vouloir donner raison au professeur Lépine, même s'il concède que l'on puisse «si nous recherchons les origines historiques de la traduction scientifique... les trouver... chez Voltaire", qui, pendant son exil en Angleterre (1726-28), traduisit l'Introduction à la Physique de Newton.

Et pourtant! C'est bien plus loin dans le temps qu'il nous faut remonter pour trouver ces origines. Le moment est venu de rendre à la traduction scientifique ses lettres d'ancienneté, qui ne le cèdent en rien à celles de la traduction

1. P. Lépine (1968): «Souvenirs d'un traducteur», Traduire, $\mathrm{n}^{\circ} 55, \mathrm{p} .21-26$

2. R. W. Jumpelt (1961): Die Uebersetzung naturwissenschaftlicher und technischer Literatur, Langenscheidt, Berlin. 
littéraire. Et pour ne point nous égarer dans le dédale des siècles et des disciplines, nous nous limiterons ici volontairement au seul domaine de la médecine.

Souvenons-nous tout d'abord que notre connaissance de la médecine hippocratique nous est venue par les Arabes. Au IX ${ }^{\mathrm{e}}$ siècle, le calife Al-Mamoun fondait à Bagdad, sous le nom de Bayt Alikhma (Maison de la Sagesse), un collège de traducteurs des ceuvres scientifiques grecques en arabe. Son premier administrateur fut le médecin personnel du calife, Jaia ben Masuiah (Mesué le Vieux), érudit nestorien formé aux sources médicales grecques et romaines. Il eut pour disciple le plus illustre, Hunayn Ibn Ishaq (809-877), connu sous les noms de Hunainus ou Johannitus, qui avec son fils Ishaq (†910), son neveu Hubayih et de nombreux élèves, traduisit en syriaque ou en arabe plus de 200 volumes, parmi lesquels le Corpus hippocraticum complet, les Aphorismes d'Hippocrate, le De differentiis febrium, le De Demonstratione, le De Sectis et l'Ars parva de Galien, la Materia Medica de Dioscoridès, etc. Cette activité traduisante a été à l'origine de la création de nombreux équivalents arabes de termes techniques.

C'est à partir de ces versions arabes que se feront par la suite les premières traductions latines. Gariopontus, de l'école salernitaine, est l'auteur d'un Passionarius Galeni ( \pm 1050 ), traduction latine d'une compilation des auteurs médicaux anciens. Constantin l'Africain (1015-1087), né à Carthage et qui enseigna aussi à l'école de médecine de Salerne, mit en latin les textes arabes des œuvres d'Hippocrate, de Galien et d'autres; avec son élève Johannes Afflacius ou Saracenus (1040-1160), il traduisit aussi, sous le titre de Pantegni (Tout l'art), le Livre Royal ou Kitab al-Maliki d'Haly Abbas, dit le Mage. Étienne le Philosophe (Antioche, début $\mathrm{XII}^{\mathrm{e}}$ ) traduisit le même ouvrage sous le nom de Liber Regius (1127), imprimé à Venise en 1492. Tous deux s'efforcèrent de substituer des mots latins, anciens ou forgés, aux termes scientifiques arabes.

Lorsque l'évêque Raymond de Tolède créa son Colegio de Traductores (1135) pour faire traduire en latin les innombrables manuscrits trouvés dans les bibliothèques arabes libérées par la Reconquista, il y attira des érudits de tout l'Occident. L'un des plus célèbres parmi eux, Gérard de Crémone (1114-1187), s'y distingua par de nombreuses traductions médicales. À côté de l'Ars parva de Galien, qu'il traduisit sur la version de Hunayn, il s'attaqua aussi aux grands de la médecine arabe et mit ainsi en latin le Canon d'Avicenne, les dix livres du second traité de Rhazès, le Kitab al-Mansouri, sous le titre de Liber Almansoris, le trentième livre de l'encyclopédie d'Abulcasis, le Kitab al-Tasrif, sous le titre de Liber Alsaharari de cirurgia, etc. Ses traductions ont conservé tels quels les termes techniques arabes, car l'arabe était alors encore parlé couramment dans les grands centres de savoir qu'étaient Tolède et Cordoue. C'est d'ailleurs dans cette dernière ville que naquit Abn Imram Musa ben Maimun (1135-1204), médecin juif espagnol mieux connu sous le nom de Maimonides, et traducteur lui aussi du Canon d'Avicenne, mais en hébreu ${ }^{3}$. Les Juifs ont en maintes occasions rempli ce rôle d'intermédiaire, tel ce Farag ben Salem, traducteur au service de Charles d'Anjou, qui donna le premier livre de Rhazès en latin sous le titre de Continens (1279).

3. La dernière traduction latine du Canon d'Avicenne fut l'œuvre de Plempius, Louvain, 1658. 
Pour voir paraître la première restitution de Galien directement du grec en latin, il fallut attendre le xive siècle. Nicolo da Reggio (1280-1350) dédia à Robert d'Anjou, roi de Naples, son De Usu partium corporis humani (1322), traduit «de verbo ad verbum, nihil addens, minnens vel parmutans $»$ sans se préoccuper des versions arabes antérieures. Il en est de même des neuf premiers exposés de médecine traduits de Galien par Guy de Parme dans son Liber notabilium (1345) composé pour Philippe VI de Valois. La traduction du De motu musculorum, faite en 1522 par Leoniceno (1428-1524), introduisit définitivement le galénisme dans la médecine occidentale.

Les traductions latines se multiplièrent ensuite pour embrasser les auteurs les plus divers. En Italie, le médecin Georges Valla traduisit l'Onomastion en dix livres de Julius Pollux (134-192) sous le titre de De humani corporis partibus (1527). Aux Pays-Bas, Jan Winter, de son nom latinisé Johannes Guinterus, donna la $\mathrm{De} R e$ Medica de Paul d'Egine, traduction dont Rembert Dodoens (1517-1585), connu aussi sous le nom de Dodonée, médecin et botaniste à Malines, publia une version révisée sous le titre de Paulus Aegineta, a Joanno Guintero latine conversus, a Remberto Dodonaeo ad graecum textum accurate collatus ac recensitus (1546). Jean-Günther d'Andernach (1505-1574), d'abord professeur de latin et de grec à Louvain, puis docteur-régent à Paris, traduisit les ouvres de Galien. Au Mexique, Juan Badiano, scribe indigène, mit en latin les parties médicales du codex d'un médecin indien sous le titre de Libellus de medicinalibus Indorum Herbio (1552). En Espagne, le médecin Andrés Laguna fit paraître à Valence sa traduction de l'œuvre du maitre de la pharmacologie grecque, Dioscorides Pediano, sous le titre de De materia medica. De universa medica (1596). Mais l'un des plus prolifiques traducteurs médicaux de l'époque fut sans conteste le médecin et botaniste Charles de l'Escluse ou Clusius (15261609), originaire d'Arras et mort à Leiden après avoir parcouru l'Allemagne, l'Autriche, la Hongrie, l'Espagne, le Portugal, l'Angleterre, la Suisse et les Pays-Bas. Polyglotte remarquable, il maniait avec aisance un latin élégant, le grec, le français, le flamand, l'anglais, l'allemand, l'italien, l'espagnol, le portugais. On lui doit ainsi des traductions latines de diverses langues: de l'italien, il a donné le Ricettario, pharmacopée florentine, sous le titre de Antidotarium, sive de exactae componendorum miscendorumque medicamentorum ratione libri tres (1561); du portugais, il a traduit Coloquios dos Simples de Garcia de Orta sous le titre de Aromatum et simplicium aliquot medicamentorum apud Indos nascentium Historia (1567); de l'espagnol, il nous a laissé deux ouvrages, le premier de Nicolas de Monardes sous le titre de De Simplicibus Medicamentis ex Occidentali India delatis, quorum in Medicina usus est (1574), le second de Christoval Costa sous le titre de Aromatum et Medicamentorum in Orientali India nascentium Liber (1582). Enfin, à côté de ces traductions latines, il convient de mentionner son Histoire des Plantes (1557) «nouvellement traduite de bas Aleman en François» du Cruydeboeck (1554) publié en flamand par le médecin et botaniste malinois Dodoens.

Il va de soi que l'Escluse n'était pas le premier à avoir traduit en langue française des textes médicaux. Dès le XIII' ${ }^{\mathrm{e}}$ siècle, en effet, avait paru une traduction française de la Rogérine, du salernitain Ruggero Frugardi, le plus 
ancien traité de chirurgie occidentale. Et, assez curieusement, c'est encore la chirurgie qui va surtout intéresser les traducteurs dans les siècles suivants. Car, mis à part la traduction des Aphorismes d'Hippocrate que Martin de Saint-Gille rendit en 1362 sous le titre de Amphorismes ypocras, on relève essentiellement: une traduction du latin de la Cyrurgie dite de l'abbé Poutrel par Jean de Prouville $(\dagger 1317)$; une version incomplète et erronée de la Cyrurgia (1314) de Henri de Mondeville, écrite en latin en 1306; la première traduction française de la Chirurgia Magna de Guy de Chauliac, due à Nicolas Panis et imprimée en 1478 avant de l'être en latin; la première traduction française de la Practica (ou Chirurgia Magna) de l'Italien Lafranchi, publiée en 1479 par le chirurgien lyonnais Guillaume Yvoire; une traduction par Nicolas Prévost de la Cyrurgia (1492) de Guillaume de Saliceto; des traductions des œuvres du célèbre chirurgien calabrais Bruno de Longoburgo publiées à partir de 1498. La Practica de fistulo in ano, du chirurgien médiéval anglais John Arderne (1307-1377) fut, elle aussi, traduite en français dès le $\mathrm{x} v^{e}$ siècle.

Le même phénomène se prolongea au $\mathrm{XVI}^{\mathrm{e}}$ siècle, et si l'on trouve bien quelques traductions comme l'Épitomé ou abrégé des trois premiers livres : de la composition des médicaments (1545) et les Aphorismes (1552) d'Hippocrate dues à un juriste du nom de Jean Brêche, ou comme l'Anatomie (1569) de Vésale par le poète et médecin Jacques Grévin (1538-1570), ce sont encore les ouvrages chirurgicaux qui se taillèrent la part du lion. Nicolas Godin adapta en français la Practica in arte chirurgica copiosa (1514), de Giovanni de Vigo, sous le titre de De Vigo en françoys, sensuit la practique et cirurgie (1525). Une traduction française de l'ouvrage écrit en latin par Jean Tagault, doyen de la Faculté de médecine de Paris, De chirurgica institutione libri quinque (1543), parut à Lyon en 1549. De nouvelles adaptations de la Chirurgia Magna virent le jour sous la plume de Jean Canapé en 1558, de Laurent Joubert (1529-1583) en 1585. Entretemps, la Chirurgia (1544) de l'humaniste et chirurgien italien Vidius avait été traduite du latin en 1565, tandis que Die grosse Wundartzney (1576) un traité chirurgical de Paracelse, l'était de l'allemand en 1589.

L'attrait de la chirurgie sur les traducteurs médicaux continua de s'exercer tout au long du $\mathrm{XVII}^{\mathrm{e}}$ siècle. En 1628 parut la traduction française de l'ouvrage rédigé en latin par Pierre Pigray, chirurgien de Henri IX et de Louis XIII, Epitome praeceptorum medicinae chirurgicae (1612); en 1669, celle de la Chirurgia (1601), écrite elle aussi en latin par Jean Riolan; en 1675, celle de l'Armamentarium chirurgicum (1655), célèbre traité sur les instruments chirurgicaux de l'Allemand Johann Schultheis, dit Scultetus, sous le titre Arsenal de Chirurgie; en 1689, celle de la Practica der Wundartzney (1563) du chirurgien suisse Felix Würtz. À côté de ces ouvrages chirurgicaux, il est vrai, on note diverses traductions venues des horizons les plus variés, telle celle publiée en 1619 par Antoine Colin du Tratado de las drogas y medicinas de las Indias orientales, con sus plantas (1578), du médecin et naturaliste portugais Christophe Acosta (15151580); ou celle parue en 1668 du De efficaci medicina libri III, dans lequel le médecin italien Marc-Aurèle Severini (1580-1656) traitait de l'emploi du feu; ou celle encore du premier ouvrage chinois, le Mö-king, traité de physiologie classique de Wang Chou-ho (280 après J.C.) que le Révérend Père Hervieu traduisit 
en 1671 sur la version latine publiée antérieurement par le Révérend Père Boym (1612-1659).

Au siècle suivant, cette même version latine servit à une nouvelle adaptation française (1736) du jésuite Jean-Baptiste du Halde (1674-1743), lequel traduisit aussi partiellement le Pen-ts'ao-kang-mou (Traité de matière médicale, 1731) de Li Che-tchen (1518-1593), célèbre pharmacologue de la dynastie Ming. Le XVIII ${ }^{\mathrm{e}}$ siècle vit ainsi se diversifier davantage et les pays d'origine et les orientations des ouvrages traduits. En 1724, le chirurgien parisien Jean Devaux traduisit l'Abrégé anatomique de Maistre Laurent Heister, le célèbre anatomiste allemand. En 1734 parut à Paris la traduction française des Operationes chirurgicae novum lumen exhibentes obstetricantibus (1701), du Hollandais Hendrik van Deventer. Quelques années plus tard, en 1741, ce fut au tour du Treatise on the operations of surgery (1737) du chirurgien anglais Samuel Sharp. MarcAntoine Eidous traduisit, de l'anglais également, un Traité des fièvres (1746) et le Dictionnaire universel de médecine (1746) de Robert James, ce dernier ouvrage en collaboration avec Diderot. Le chirurgien français Toussaint Bordenave (1728-1782) mit en français le traité du physiologiste suisse Albert de Haller Elementa physiologiae (1757-66). La chirurgie se manifesta encore avec la publication, en 1789, d'une traduction du Trattato delle operazioni di chirurgia (1763) du professeur turinois Giovanni Bertrandi; celle, en 1770, de la traduction en trois volumes des Institutiones chirurgicae (1739) de Lorenz Heister, fondateur de la chirurgie scientifique allemande, sous le titre de Institutions de chirurgie, où l'on traite dans un ordre clair et nouveau ce qui a rapport à cet art, ouvrage de quarante ans, traduit du latin de M. Laurent Heister; et celle, en 1796, des six volumes de $A$ system of surgery (1782-87) du chirurgien anglais Benjamin Bell. Mais la fin du siècle vit les traducteurs s'intéresser aussi à un Traité sur la génération des vers intestinaux et sur les moyens de les détruire (1772), traduit du médecin et naturaliste allemand Mark Bloch en 1788; à un traité sur les hernies, Abhandlung von den Brüchen (1777-79), traduit du chirurgien allemand August Richter en 1788 également; aux Éléments de l'art des accouchements de Josef Plenck, traduits par J. Pitt en 1789; aux Recherches sur les causes et les effets de la variole, du célèbre médecin anglais Edward Jenner, dont la traduction parut en 1798.

La démonstration de l'ancienneté de la traduction scientifique - caractérisée en l'occurrence par la traduction médicale - pourrait s'arrêter ici. Manifestement, ses origines sont de loin antérieures à Voltaire. Pour être complet, cependant, il conviendrait encore de montrer qu'il en est ainsi pour d'autres langues occidentales et que, même en français, de nombreuses traductions ont encore vu le jour avant même le $\mathrm{xx}^{\mathrm{e}}$ siècle. Elles peuvent se répartir en trois tendances. À la première appartiennent les versions de textes anciens déjà traduits antérieurement ou traduits pour la première fois. C'est ainsi qu'il se trouve un Émile Littré, lexicographe et médecin, pour entreprendre une nouvelle traduction des Oeuvres d'Hippocrate (1839-61). En 1854, Jean Carolus d'Anvers mit en français moderne les trois premiers chapitres du Traité de chirurgie (1310) de Jean Yperman. En 1893, la Cyrurgia (1314) de Henri de Mondeville fut retraduite en français moderne par E. Nicaise, en collaboration avec M. F. Cha- 
vannes et le $\mathrm{D}^{\mathrm{r}}$ Saint-Larger. Et quelques années plus tard, en 1898, Paul Pifteau fit de même pour la Cyrurgia de Guillaume de Saliceto, sous le titre de Chirurgie de Guillaume de Salicet. Auparavant, Boucher avait donné la première traduction française du De praxi medica ad priscam observandi rationem revocanda (1696) du médecin italien Georgio Baglivi, sous le titre De l'accroissement de la médecine pratique (1851). La deuxième tendance est représentée par des traductions d'ouvrages exotiques - chinois, japonais, arabes. Plusieurs chapitres importants du Pen-ts'ao furent ainsi traduits par Geerts (1843-1883), tandis que le $\mathrm{D}^{\mathrm{r}}$ Albert Husson mettait en français le Tchen-kieou ta-tch'eng (1579), traité d'acupuncture et d'ophtalmologie du grand maître de l'époque Ming, Yang ki-tcheou. Pour le domaine japonais, le $\mathrm{D}^{\mathrm{r}}$ Charpentier fit paraître en 1879 une traduction du San-ron (1765), de l'obstétricien Kagawa Genetsu, et une autre de l'Obstétrique au Japon de B. Miyake. En 1896, F. de Mély (18511935) traduisit divers chapitres de l'encyclopédie Ho-han san-ts'ai t'ou-houei, qui reproduisent en grande partie les chapitres correspondants du Pen-ts'ao kang-mou. Quant au domaine arabe, de nouvelles versions du Kitab-al-Mansouri de Rhazès, du Quarrum de Haly Abbas, et du Kitab-al-Maliki d'Avicenne virent le jour sous la plume de P. de Koning (1903) et M. Simon (1906). A la troisième tendance, enfin, ressortissent les traductions d'ouvrages contemporains, comme celle du premier traité systématique anglais d'anatomie pathologique, The morbid anatomy (1793), de Matthew Baillie, publiée en 1803. De l'allemand, A.J.L. Jourdan traduisit la Macrobiotique, ou l'art de prolonger la vie de l'homme, suivi de conseils sur l'éducation physique des enfants (1838) de Christoph Hufeland, ainsi que l'Histoire de la médecine depuis son origine jusqu'au XIX ${ }^{e}$ siècle (1815-20) de Kurt Sprengel. En 1878 parut, sous le titre de les Nerfs et les muscles, la traduction d'un ouvrage du physiologiste allemand Isidor Rosenthal publié en 1877. En 1880, le $\mathrm{D}^{\mathrm{r}}$ A.H. Robin traduisit le Traité d'embryologie et d'organogénie comparées du physiologiste anglais Maitland Balfour, paru en 1875. Quelques années plus tard, F. J. Herrgott publia sa traduction en trois volumes de l'Essai d'une histoire de l'obstétricie (1891-92) de l'Allemand Ed. von Siebold.

Examinons à présent brièvement ce qu'a été l'évolution de la traduction médicale dans quelques autres langues occidentales. En Grande-Bretagne, où l'anglais ne fut reconnu comme langue officielle qu'en 1399, date à laquelle le roi Henri IV en usa pour la première fois au Parlement, c'est d'emblée que l'on enregistra une traduction médicale du livre du médecin et botaniste espagnol Nicolas de Monardes sur les plantes médicinales du Nouveau Monde; elle était l'œuvre de John Frampton, qui l'intitula Joyfull Newes out of the newe founde Worlde. En 1539, le médecin-diplomate Thomas Elyot traduisit les prescriptions et remèdes de Galien et d'autres médecins de 1'Antiquité dans Castel of Helth. La version intermédiaire latine, De partu hominis, de l'ouvrage Der swangeren Frauen und Hebamen Rosegarten (1513) d'Eucharius Rösslin, servit à Richard Jonas à en faire une traduction anglaise sous le titre de The Byrth of Mankynde (1540). C'est sur une version intermédiaire également, la française de l'Escluse, que Henry Lyte traduisit le Cruydeboeck de Dodoens, en l'intitulant $A$ Niewe Herball or Historie of Plantes (1578). En 1633, J. Hester fit paraitre une traduc- 
tion savoureuse de certains écrits du médecin suisse Paracelse. Les Livres de la chirurgie d'Ambroise Paré furent traduits, par le biais d'une version latine une fois de plus, par Th. Johnson sous le titre The Workes of that famous Chirurgion Ambrose Parey translated out of Latine and compared with the French (1634). Un peu plus tard, le médecin et chirurgien James Cooke mit en anglais «for common benefit» les Select Observations on English Bodies (1657) publiées «first in Latine» de John Hall. Les Observations de chirurgie (1737), dans lesquelles le chirurgien militaire français Henri Le Dran étudiait les effets des traumatismes de guerre sur la «machine animale» et créait le terme choc, furent traduites en 1743 ; on y trouve pour la première fois le terme shock dans la forme anglaise qui allait en assurer le succès. En 1801, John Hull traduisit Deux mémoires sur la césarienne du chirurgien et obstétricien Jean-Louis Baudelocque. Vers la moitié du siècle, c'est John Forbes (1781-1861) qui donna une traduction du Traité de l'Auscultation médiate de Laënnec. Enfin, au rayon des ouvrages exotiques, on pouvait noter la traduction par J. Dudgeon, en 1893-94, du Yi-lin kai-tso (Correcteur des erreurs médicales) de l'anatomiste chinois moderne Wang Ts'ing-Jen (1796-1821), ainsi que des versions partielles du Pen ts'ao kang-mou par F. Porter Smith et E. Bretschneider (1894).

En Allemagne, une traduction médicale parut déjà en 1543 : il s'agissait de la version allemande de l'Epitome de Vésale, réalisée par Alban Torinus. En 1609 , on trouve une traduction des Observations diverses sur la stérilité de l'obstétricien français Loyse Bourgeois. Elle fut suivie, en 1634, de la traduction d'un ouvrage féminin, le Catoptron microcosmicum (1613) de Johann Remmelin, publiée sous le titre de Kleiner Welt-Spiegel, et par celle, en 1668, du Traité des maladies des femmes grosses et de celles qui sont accouchées de François Mauriceau. Au siècle suivant, c'est une œuvre du fondateur de la pédiatrie moderne, le Suédois Nils Roser von Rosenstein, Underrätelser om barnjukdomar och deras botemedel (1764) qui passa dans la langue allemande (1766). Samuel Hahnemann, (1755-1843), fondateur de l'homéopathie et traducteur scientifique éminent du XviII $^{\mathrm{e}}$ siècle, publia de nombreuses traductions médicales, parmi lesquelles les Eaux minérales et les bains chauds de Falconer, la Nouvelle Façon de soigner de Boll, les Précautions à prendre par les femmes de Quigg, une Pharmacologie de Monroe et la Materia Medica de W. MacCullen. Le médecin Philipp-Franz von Siebold (1796-1866), pour sa part, traduisit le SanRon (1765) du père de l'obstétrique japonaise moderne, Kagawa Genetsu; c'est cette version qui servit à la traduction française du $\mathrm{D}^{\mathrm{r}}$ Charpentier.

En Italie, la Cyrurgia (1210) de Guillaume de Saliceto fut traduite en italien dès le xrve siècle et publiée à Venise en 1474 sous le titre la Ciroxia vulgarmente fata. En 1595, c'est Scipione Mercurio (1540-1615) qui traduisit le Rosegarten (1513) d'Eucharius Rösslin sous le titre la Comare Raccoglitrice. Une première traduction italienne du Traité de l'obstétricien Mauriceau parut avant la fin du $\mathrm{XVII}^{\mathrm{e}}$ siècle.

Aux Pays-Bas, la Cyrurgia (1306) d'Henri de Mondeville connut sa première traduction néerlandaise avant la fin du siècle. Les Vivae imagines partium corporis humani aereis formis expressae, publiées chez Plantin à Anvers, en 1566, furent traduites deux ans plus tard. En 1592, Carel Baten, de Gand, protes- 
tant réfugié à Dordrecht où il exerça la médecine de 1588 à 1601 sous le nom de Carolus Battum, traduisit la Chirurgie d'Ambroise Paré sous le titre De Chirurgie ende alle de Opera, ofte Werken van Mr. Ambroise Paré. Au XvII siècle parurent une traduction néerlandaise des Observations (1609) de Bourgeois et une autre du Traité (1668) de Mauriceau. En 1734, Gerardus Dieten traduisit un ouvrage de l'anatomiste allemand Johann Kulmus sous le titre Ontleedkundige taferelen, benevens de daartoe behoorende afbeeldingen en aanmerkingen. In het needertuitsch gebracht door Gerardus Dieten. Enfin, le début du XIX ${ }^{\mathrm{e}}$ siècle vit paraître deux traductions du japonais : la première du San-Ron (1825) déjà cité, par un traducteur japonais, Miwa Junzo; la seconde d'un traité sino-japonais sur l'acupuncture et les moxas, publiée sous le titre de Beschrijving van het naalde steeken en moxa branden (1827) par le Hollandais Isaac Titsingh (17451812).

Les nombreux exemples évoqués ici prouvent à suffisance que la traduction médicale n'est pas un phénomène récent. L'homme moderne n'a que trop tendance à croire que tout est né avec son temps et que rien n'a été fait avant lui. Certes, le rétrécissement du monde, la multiplication et l'accélération des contacts entre spécialistes grâce aux milliers de congrès qui s'organisent chaque année, l'envahissement par la traduction de tous les domaines de la culture, de la technique et de la science, peuvent renforcer cette impression de redécouvrir sans cesse le monde. Mais s'il est vrai que la traduction technico-scientifique a sans doute pris son véritable envol au $\mathrm{xx}^{\mathrm{e}}$ siècle, il nous faut reconnaître en toute humilité que, dans ce domaine comme dans beaucoup d'autres, nos prédécesseurs avaient défriché déjà un terrain considérable et fait du bon travail. 